\title{
CERTAINTY AND SUBJECTIVITY IN ENGLISH EDUCATION RESEARCH: A CROSS-CULTURAL SYSTEMIC FUNCTIONAL ANALYSIS
}

\author{
May Mahdi Alramadan \\ King Faisal University, Al-Ahsa, Saudi Arabia \\ Author Email: malramadan@kfu.edu.sa
}

\begin{abstract}
Article Info
Article History

Received: December 2021

Revised: December 2021

Published: January 2022

Keywords

Attitude;

Authorial stance;

Boosters;

Epistemic modality;

Hedgers;

Abstract

This study examines the use of epistemic modality (expressions that signal varying degrees of certainty and subjectivity) by writers of English education research. Epistemic modality is a crucial, yet intricate, rhetorical device through which writers qualify their claims and construct a stance towards their texts and readers. Disciplinary and cultural norms influence the rhetorical use of modality in academic texts. To understand the impact of these contextual factors, linguistic descriptions need to examine discourse produced in individual disciplines and even subdisciplines. Using an exploratory comparative approach, the present study analyzes education research that is produced by native-English-speaking and EFL Arab writers: (1) to describe the discipline-specific practices that are adopted by native writers to manipulate the degrees of certainty and subjectivity in their discourse; and (2) to explore how these practices vary cross-culturally. Sixty research papers are analyzed using the finely grained model of Systemic Functional Linguistics. The findings show a disciplinary preference whereby native writers avoided expressing hesitancy and doubt and preferred a moderately confident epistemic stance to create convincing arguments. These writers' epistemic style was also objective and detached. The EFL texts, in contrast, were less dialogic and had higher levels of confidence, explicitness, and subjectivity. Although advanced in their linguistic and disciplinary proficiency, the non-native writers displayed some patterns that are generally characteristic of other Arab and non-Arab L2 writers/learners, indicating the vital role that culture and nativeness play in rhetorical strategy use. The paper ends by highlighting the need for explicit instruction of epistemic modality in Arab higher education institutions in order for non-native writers to produce academic texts that are persuasively effective from the perspective of the international academic community.
\end{abstract}

How to cite: Alramadan, M. A. (2022). Certainty and subjectivity in English education research: a cross-cultural systemic functional analysis, JOLLT Journal of Languages and Language Teaching, 10(1) pp. 1-18. DOI: https://doi.org/10.33394/jollt.v\%vi\%i.4548

\section{INTRODUCTION}

It is now widely recognized that written academic discourse does not only aim to provide factual information to represent an external reality, but also involves the projection of an authorial stance aimed to persuade readers with the relevance and significance of the reported research (Hyland, 1994; Vold, 2006). Academics endevour to construct rhetorically effective texts, and in order to do so they complement their informative propositional content with their opinions, evaluations and subjective interpretations including their comments on the degree of validity and truthfulness of the information that they provide. However, writers are not free to employ the rhetorical resources that they personally see fitting. Rather, they make a strategic selection from a constrained set of socially-situated options that are conventionally found acceptable and convincing by the target discourse community (Hyland, 2005; Varttala, 2001). This means that deviation from the rhetorical norms that are maintained by the discourse community may eventually lead to inappropriate social interaction in written texts. One of the 
rhetorical features that allow writers to effectively achieve such interactional and persuasive functions si epistemic modality (Myers, 1989; Hyland \& Milton, 1997; Vold 2006; Akbas \& Hardman, 2020).

Several definitions have been provided for epistemic modality. These definitions agree on the role of modality in signaling writers' attitudes concerning the factual nature of the described situations and their personal judgment about how likely the utterances are. One of the earliest definitions was provided by Lakoff (1972, p. 195), who talked about phrases "whose job is to make things fuzzier," referring to expressions that allow writers to be less committed to the referential information of their propositions. Lyons (1977, p. 793) pointed to the distinction between fact and opinion, stating that modality is "concerned with matters of knowledge, belief, or opinion rather than fact". Modality represents a cline with varying levels of strength that enables writers to express different degrees of confidence ranging from weak possibility, It may be the case, to strong necessity, It must be the case, (Collins, 2009). Thus, modality involves two kinds of expressions: expressions that indicate the addresser's lack of commitment to the validity of the claims, or hedges (e.g., may, might, possibly), and expressions that convey the addresser's more heightened investment in the truth of the utterance, or boosters (e.g., must, certainly, indeed). Modality can be encoded in a wide range of linguistic resources, including modal verbs (may, might, could), adjectives (possible, doubtless, clear), adverbs (perhaps, certainly, usually), nouns (fact, evidence, likelihood), and lexical verbs (seem, appear, think).

Modality expresses several semantic meanings, including "possibility, necessity, ability, obligation, permission and hypotheticality" (Collins, 2009, p. 11). These functions are similar in that they involve "non-factuality" or the meaning that what is being proposed is not a straightforward, known fact (Collins, 2009, p. 11). This makes the writer's stance more reliable and precise, as the distinction between fact and opinion is clearly drawn for the reader.

Scholars have discussed the pragmatic and dialogic functions of modality in academic discourse. In this regard, modality is said to assist writers to represent their authorial stance in accordance with the degree of certainty that is used. Thus, writers can appear tentative, cautious, polite, and humble (Vold, 2006) by withholding their commitment to claims through hedges. Alternatively, they can sound confident, assertive, and authoritative (Akbas \& Hardman, 2018) through reinforcement of truthfulness by boosting resources. Also, through the manipulation of the syntactic context incorporating modality expressions, writers can manage how subjectively or objectively their epistemic attitude is imparted (Halliday \& Matthiessen, 2004). Epistemic modality also allows writers to make their discourse more interpersonal by showing that they anticipate readers' responses and, accordingly, adjust the degree of certainty of claims (Hyland, 1994). Hedges are used as a politeness strategy that minimizes the force of statements in order to save face for both the writer and reader when advancing new knowledge claims and when criticizing aspects of the literature (Myers, 1989; Vold, 2006). Boosters, on the other hand, serve to invoke common disciplinary knowledge and join participants in shared communities (Myers, 1989). Additionally, modality performs dialogic, alignment, and reader-engagement functions by either contracting or expanding the dialogic space for alternative value positions (Martin \& White, 2005).

Existing research recognizes the critical role of epistemic modality in academic discourse (Hyland, 1996; Takimoto, 2015; Dogan and Akbas, 2021; Farnia and Gerami, 2021). It also suggests the impact of disciplinary and cultural factors on the nature of the employed epistemic resources (Hyland \& Milton, 1997; Koutsantoni, 2005; Akbas \& Hardman, 2018). However, there still remains a paucity of evidence on the detailed character of the epistemic stance that is adopted by native English-speaking writers in specific academic disciplines especially if this stance is compared to the one exhibited by non-native writers. This is significant to understand if accurate characterizations of discipline-specific rhetorical styles are to be determined to serve 
both descriptive and pedagogical purposes. To this end, the present study adopts the detailed framework offered by Systemic Functional Grammar (Halliday \& Matthiessen, 2004) to describe the epistemic stance that is employed by native writers of education research and to examine whether the comparative stance exhibited by expert non-native writers conforms to or detaches from native norms and conventions.

\section{Literature Review}

A wealth of studies has accumulated on the use of epistemic modality in academic discourse. Evidence shows that epistemic modality is crucial in English academic discourse (Hyland, 1994; Hu \& Li, 2015) and that its use is associated with the quality and acceptance of the produced texts (Hyland \& Milton, 1997; Akbas \& Hardman, 2018). Additionally, modality is a complex linguistic system, and this makes non-native writers struggle with managing its nuanced dimensions (Holmes, 1982; Vold, 2006).

Modality studies have adopted two methodologies that are sometimes used simultaneously: examining how writers use modality with different linguistic/cultural backgrounds, and describing the use of modality in different academic disciplines. Researchers conducting culture-based comparisons acknowledged the role that culture plays in the use of modality, with writers coming from different cultural backgrounds preferring to use different modality degrees and wordings. This research has indicated the tentative stance that Englishspeaking academics normally opt for, especially when compared to writers from other cultures (Hyland \& Milton, 1997; Koutsantoni, 2005; Akbas \& Hardman, 2018). Vold (2006) found that culture was more important than discipline in differentiating between the epistemic styles used by English, Norwegian, and French academics, with French writers preferring a more confident and assertive style than their counterparts. In a similar vein, Akbas and Hardman (2018) revealed the important role of culture/language in leading English-speaking and Turkish EFL writers to project a more tentative stance than Turkish L1 writers. Researchers attributed the difference between the two Turkish groups to the Turkish EFL writers' awareness of the English modality system and their self-development on how to use it properly. With regard to Arab EFL writers, research has pointed to the tendency to assume a relatively authoritative and confident stance as a result of the scarcity of interactional strategies (Al-Mudhaffari et al., 2020) and the expression of certainty through exaggerating expressions (Abu Rass, 2011), emphatics (ElSeid, 2000), boosters (Sultan, 2011), and more monoglossic and dialogically contractive resources (Alramadan, 2020b). However, although the current Arab-based evidence is informative, it largely focuses on learner writers, both graduate and undergraduate. Fewer studies exist (e.g, Sultan, 2011; Alotaibi, 2015; Alramadan, 2020b) that tackle how expert Arab EFL writers employ hedges and boosters in a way deemed conventional by the target discourse community. Furthermore, even in these studies, modality was not investigated as the target feature that comprises a comprehensive system of meaning-making but was rather included as one of the aspects in the overall reader-engagement system that was employed in texts. Further research is therefore needed to focus on Arab expert writers' use of the modality system specifically to provide more detailed descriptions of their epistemic stance in different genrespecific and discipline-specific contexts and to reveal how this stance compares to native norms and conventions.

Discipline-based studies point to discipline, too, as an important factor constraining the nature of authorial epistemic stance. Certainty and commitment were investigated in various disciplines, including medicine (Salager-Meyer, 1994; Yang et al., 2015), science (Butler, 1990), dentistry (Crosthwaite et al. ,2017), engineering (Koutsantoni, 2005), computer science (Mirzapour \& Mahand, 2012), and applied linguistics (Vold, 2006; Al-Mudhaffari et al., 2020). Hyland (1998) explored eight disciplines in humanities, social sciences, and natural sciences revealing the tendency of discourse in the natural sciences to use fewer interactional resources 
and to emphasize objectivity to a greater degree than discourse in the humanities and social sciences. Generally, this scholarship showed that there is a "considerable variation in disciplinary knowledge-making practices" (Hyland, 1998: 354), with writers' choices not only reflecting their own individual positions but also the epistemological beliefs and values of the community (Crosthwaite et al., 2017). Vold (2006) argued that every discipline seems to have its own preferred rhetorical strategies and, to understand disciplinary variation fully, "other disciplines and even subdisciplines need to be compared in future research" (p. 80) because "results from one discipline cannot automatically be transferred to other disciplines" (p. 63). One of the disciplines that, apparently, have not been subjected to linguistic analysis is the discipline of education.

In relation to the analytical frameworks employed in the literature, the majority of modality studies tended to investigate the employment of a list of epistemic markers that were compiled by the researcher(s) based on pilot explorations (e.g., Vold, 2006) or that were part of analytical frameworks proposed in the literature, such as the work of Holmes (1984), Hyland (1996), and Hyland and Milton (1997). Very few studies (e.g., Yang et al., 2015) analyzed the use of modality from a Systemic Functional Linguistics perspective (SFL; Halliday \& Matthiessen, 2004). A couple of features make the SFL framework more comprehensive and analytically powerful. First, the former frameworks rely on a binary classification of markers, where expressions function as either hedges or boosters. The SFL framework, in contrast, allows a more nuanced analysis that classifies markers according to a three-point system: low, median, and high. Undoubtedly, more detailed analyses would be more insightful from descriptive as well as pedagogical perspectives. Second, only the SFL framework consolidates values of modality with orientations of modality - that is, the notion of certainty with the notions of explicitness and subjectivity. It does so through a consideration of the syntactic structures that incorporate the used epistemic lexemes. Indeed, this is an important feature to utilize because the construction of effective authorial stance not only involves the use of the correct degree of certainty but also the correct degree of explicitness and subjectivity that is expected by the target discourse community (Hyland, 2016).

\section{Research Questions}

Using an SFL perspective, the present study investigates the notions of certainty and subjectivity and compares their employment in the discourse that is produced by nativeEnglish-speaking (NS) and non-native-speaking (NNS) Saudi Arabian professional writers. Sixty research articles in education are analyzed. The study answers the following questions: (1) What degree of epistemic certainty characterizes the stance taken by writers in the field of education? (2) How subjectively are the epistemic attitudes imparted in this field? and (3) How do English and Arabic-speaking EFL writers differ in their use of epistemic stance?

\section{Theoretical Framework: Modality in SFL}

In SFL, modality is defined as an expression of indeterminacy (Halliday \& Matthiessen, 2004). By using modal expressions, speakers convey their lack of certainty in the validity of the information. Modals fall into systems of types and scales. With regard to the types of modality, different speech roles require distinct types of modality, thus, SFL differentiates between modalization and modulation. Modalization is related to propositions (or the exchange of information as a commodity) and it involves the use of epistemic expressions to signal the degree of confidence that the speaker has about the validity of the presented attitude in terms of both its likelihood (how probable it is to be true: possible/probable/certain) and its usuality (how often it is true: sometimes/often/always). On the other hand, modulation regards proposals (or the exchange of goods and services) and it involves the expression of different degrees of obligation on the other person to carry out commands (e.g., permissible/advisable/obligatory), 
and the expression of different degrees of willingness or inclination on the part of the speaker to fulfill an offer (e.g., ability/willingness/determination; Thompson, 2014, pp. 69-71).

Each modal expression signals a specific degree along two simultaneous scales: value and orientation. The scale of value indicates the strength of a writer's commitment to the truth of utterances. A writer's certainty can be high ("I shall never be happy again"), median ("They should be back by now"), and low ("I may be quite wrong"; Thompson, 2014). The scale of orientation expresses the degree to which the speaker is ready to assume overt responsibility for the presented judgment. There are four points along the scale of responsibility. Table 1 illustrates these degrees, with examples taken from the present data. The model expressions are in bold.

Table 1.

Orientations of modality

\begin{tabular}{lll}
\hline \multicolumn{1}{c}{ Orientation } & \multicolumn{1}{c}{ Structure } & Example \\
\hline Explicit subjective & Projecting mental clause & $\begin{array}{l}\text { "I believe that policy makers have made } \\
\text { progress" }\end{array}$ \\
\hline Implicit subjective & Modal operators & $\begin{array}{l}\text { "Giving these students an opportunity to use } \\
\text { their prior knowledge might have been a bridge" }\end{array}$ \\
\hline Implicit objective & $\begin{array}{l}\text { Epistemic adjectives, adverbs, } \\
\text { nouns, verbs }\end{array}$ & $\begin{array}{l}\text { "Perhaps the most vivid example has been in } \\
\text { England." }\end{array}$ \\
\hline Explicit objective & Projecting relational clause & $\begin{array}{l}\text { "It is evident from their classroom observation } \\
\text { that they teach in exactly the same way they } \\
\text { were taught." }\end{array}$ \\
\hline
\end{tabular}

The four orientations differ from each other in their syntactic makeup. Modality in implicit orientations is expressed in the clause containing the proposition. In explicit orientations, modality is expressed in a clause of its own. The four orientations allow different degrees of authorial responsibility. The orientation with the highest degree of responsibility is the explicit subjective orientation, I believe, which overtly communicates the subjectivity of the claim. This orientation signals that the writer is the direct source of the conviction through the use of first-person subject pronouns I/we as the subject of the modality clause.

The implicit subjective category employs modal operators, will, may, might, to ground the statement within the writer's subjectivity and to indicate that they are the source of the assessment. However, the subjectivity encoded by this category is implicit because it is not overtly highlighted through a projecting clause and because this category, instead, "steers the reader's focus to the objective state of the proposition" (Yang et al., 2015, p. 6). The implicit objective orientation is expressed through different grammatical categories: adjectives (likely/ possible), adverbs (perhaps/ probably), verbs (seem/ appear), nouns (possibility /likelihood). This orientation leaves implicit the source of the conviction. It is "a way of objectifying the speaker's evaluation" (Halliday \& Matthiessen, 2004, p. 150) by suggesting that the assessed likelihood is an objective event rather than being an opinion that results from the addresser as the source of the evaluation.

Finally, with explicit objective resources, it's likely/possible/evident that, writers do not overtly show that they are the source of the modality, but they make their evaluations explicitly appear as an objective event about which they are simply informing the addressee (Yang et al., 2015). These constructions help to minimize writers' visibility and their role in interpreting data by construing the facts as speaking for themselves (Hyland, 1998). The modality in this orientation is not included in the clause containing the modalized proposition, but is embedded in a separate clause, mostly with a dummy it or an inanimate subject replacing a human agent. The overall system of modality is summarized in Table 2 . 
Table 2.

The modality system in SFL

\begin{tabular}{|c|c|c|c|}
\hline \multirow{11}{*}{ Modality } & \multirow{4}{*}{ Types } & \multirow[t]{2}{*}{ Modalization } & Probability \\
\hline & & & Usuality \\
\hline & & \multirow[t]{2}{*}{ Modulation } & Obligation \\
\hline & & & Inclination \\
\hline & \multirow{7}{*}{ Scales } & \multirow{3}{*}{$\begin{array}{l}\text { Value } \\
\text { (commitment) }\end{array}$} & High \\
\hline & & & Median \\
\hline & & & Low \\
\hline & & \multirow{4}{*}{$\begin{array}{l}\text { Orientation } \\
\text { (responsibility) }\end{array}$} & Explicit subjective \\
\hline & & & Implicit subjective \\
\hline & & & Implicit objective \\
\hline & & & Explicit objective \\
\hline
\end{tabular}

\section{RESEARCH METHOD \\ Methodological Approach}

The present exploratory study uses the descriptive model offered by Systemic Functional Linguistics (Halliday \& Matthiessen, 2004). This approach systematically relates language structures to language functions, and it assumes that linguistic semiotic systems, like the system of modality, are used by participants to achieve the process of meaning making by choice (Martin and White, 2005). Thus, the choices exhibited by academic writers are assumed to be strategically made to construe certain tenor configurations that are conventionally appropriate in their disciplinary and cultural contexts of discourse production.

\section{The Corpora and Subjects}

The data consist of 60 research articles written by two groups of writers: NS and NNS of English. Following previous studies (e.g., Yang et al., 2015), the nativeness of authors was ascertained through their names and university affiliation. Further details about the two corpora are provided in Table 3. All the articles belong to the academic discipline of education and particularly to the subdiscipline of curriculum and teaching methodology. These articles tackle issues related to teacher preparation, training and professional development, and theories and methods of teaching different school and university subjects.

Table 3.

Description of corpora

\begin{tabular}{lrcr}
\hline \multicolumn{1}{c}{ Corpus } & No. of texts & Date of publication & Word count \\
\hline NS & 30 & $2010-2020$ & 250,752 \\
\hline NNS & 30 & $2010-2020$ & 162,511 \\
\hline Total & 60 & & 413,263 \\
\hline
\end{tabular}

The NS articles were retrieved from the journal Teaching and Teacher Education, which is an international Scopus-indexed journal of research and empirical studies. The choice of this journal was based on convenience, as this journal is open-access, which made it easier to obtain the articles. The collection of the NNS corpus, however, required a more extended method. First, the websites of all Saudi universities were visited to find academics who work in colleges of education and, specifically, in departments of teaching methodology in these universities. Then, the curriculum vitae of each faculty member was read to obtain information about their 
English publications. Each identified research paper was then retrieved, either from online search engines or via the Saudi Digital Library. The NNS articles were published in peerreviewed national and international journals. Due to the difficulty of finding papers written by Saudi scholars in English, sampling had to cover papers published throughout an extended period of time, from 2010 to 2020 . To make comparisons valid, this sample was matched with a corresponding sample of research papers that were published by native English speakers during the same period.

It is also worth noting that all the Saudi writers hold a Ph.D. in education, and they work as assistant or associate professors in their departments. This indicates their advanced level of English-language proficiency as well as their high academic expertise and professional background in the discipline under investigation.

\section{Selection and Classification of Markers}

A list of 94 targeted epistemic markers was compiled by referring to the literature. Specifically, Halliday and Matthiessen (2004) and Thompson (2014) were consulted to prepare a list of epistemic and deontic modal expressions and to determine their degree of certainty. Hyland and Milton (1997) and Flowerdew (2002) were consulted to specify the nouns, adjective, adverbs, and verbs that are most frequently used in academic discourse. The markers were then classified according to the modality system conceptualized by Halliday and Matthiessen (2004), as described in Section 2.

The following criteria were used in order to ascertain that each identified instance functioned as an epistemic marker.

1. The semantic meaning of each marker was taken as an important indicator of its epistemic function. However, where the semantic meaning alone did not lead to a conclusive decision concerning the nature of the marker, the following two tests were used;

2. A deletion test: whereby a polysemic marker is deleted from the sentence. If the deletion results in a seemingly factual statement which is either categorically positive or negative, then the marker would have been taken as a resource for the qualification of the sentence;

3. A substitution test (following Vold et al., 2006, 72): whereby a polysemic marker of a certain value is substituted with a marker of a varying value of certainty, either higher or lower. If the substitution results in a different degree of certainty, whether higher or lower, the marker would have been taken as a signal of epistemic meaning.

\section{Research Design}

Following previous research (e.g., Vold, 2006), the study adopts a holistic approach of analyzing entire articles and not sections of them. This was done because research papers represent a distinct genre with a specific communicative function (Swales, 1990). This approach was also analytically useful because it provided a wider linguistic cross-section that ensured the inclusion of as many instances of epistemic markers as possible.

The texts were analyzed using the software WordSmith Tools (version 6), which is a freeware text-retrieval program. The material included the body of the article or its running text. The abstracts, tables, figures, appendices, bibliographies, and notes were excluded. After preparation of the corpora, a search for each of the previously identified linguistic markers in the texts was run. All the occurrences were concordanced and qualitatively analyzed within sentential context to ascertain their epistemic functions. However, the analysis not only counted the frequency of markers as separate lexemes, but also investigated the syntactic structures incorporating them in order to differentiate between the implicit and explicit orientations of modality. Following previous research (e.g., Hyland and Milton, 1997; Yang et al., 2015) 
descriptive statistics were used to compare between the two groups. Furthermore, the frequencies were normalized to occurrences per 10,000 words to allow for an accurate comparison between corpora of different sizes. The normalization process was calculated using the following equation:

$$
\text { normalized frequency }(f n)=\frac{\text { frequency of epistemic marker }}{\text { number of words in the corpus }} \times 10,000
$$

\section{RESEARCH FINDINGS AND DISCUSSION Research Findings}

The analysis revealed important observations concerning the epistemic nature of the target discipline as well as differences between the two corpora involved. The following sections display figures related to the employment of values and orientations of modality.

\section{Values of Epistemic Markers}

As shown in Table 4, the NS corpus encoded an overall larger number of epistemic markers (82.2) than the NNS corpus (77.5). This indicates the NS writers' greater tendency to qualify claims for certainty.

Table 4.

Frequency of modality values (per 10,000 words)

\begin{tabular}{|c|c|c|c|c|c|c|c|}
\hline & \multicolumn{2}{|l|}{ Low } & \multicolumn{2}{|c|}{ Median } & \multicolumn{2}{|l|}{ High } & \multirow[t]{2}{*}{ Total } \\
\hline & $f \mathbf{n}$ & $\%$ & $f n$ & $\%$ & $f \mathbf{n}$ & $\%$ & \\
\hline NS & 22.3 & $27.2 \%$ & 43.3 & $52.6 \%$ & 16.5 & $20.0 \%$ & 82.2 \\
\hline NNS & 15.9 & $20.5 \%$ & 43.6 & $56.3 \%$ & 17.6 & $23.1 \%$ & 77.5 \\
\hline
\end{tabular}

Table 2 also shows the frequencies of the different values of modality. The most frequently used value in both corpora was the median value, or probability, with more than $50 \%$ reliance on this resource. The frequency of the low category was smaller (27\% for NS and $20 \%$ for NNS writers). Additionally, there are differences between the NS and NNS groups in the frequencies of the low and high values of certainty. The NS discourse encoded more possibility markers (22.3) than the NNS group (15.9). On the other hand, the NNS writers encoded more high-value markers (17.6) than did NS writers (16.5).

\section{Orientation of Epistemic Markers}

As shown in Table 5, the two corpora are similar in the distribution of marker orientations. The implicit orientation occurs considerably more frequently than the explicit orientation in the two corpora. However, while the explicit orientation has been relatively infrequent in both corpora, it was used more often by the NNS.

Table 5.

Frequency of modality orientations (per 10,000 words)

\begin{tabular}{|c|c|c|c|c|c|c|c|}
\hline \multicolumn{4}{|c|}{ NS } & \multicolumn{4}{|c|}{ NNS } \\
\hline $\begin{array}{l}\text { Implicit } \\
\text { subjective }\end{array}$ & $\begin{array}{l}\text { Implicit } \\
\text { objective }\end{array}$ & $\begin{array}{l}\text { Explicit } \\
\text { subjective }\end{array}$ & $\begin{array}{l}\text { Explicit } \\
\text { objective }\end{array}$ & $\begin{array}{l}\text { Implicit } \\
\text { subjective }\end{array}$ & $\begin{array}{l}\text { Implicit } \\
\text { objective }\end{array}$ & $\begin{array}{l}\text { Explicit } \\
\text { subjective }\end{array}$ & $\begin{array}{l}\text { Explicit } \\
\text { objective }\end{array}$ \\
\hline 53.6 & 24 & 1.3 & 3.1 & 56.8 & 14.3 & 1.5 & 4.7 \\
\hline $65 \%$ & $29 \%$ & $1.6 \%$ & $3.8 \%$ & $73 \%$ & $18 \%$ & $2 \%$ & $6.1 \%$ \\
\hline
\end{tabular}

The most frequently used orientation is implicit subjective (might, can, could), with 53.6 occurrences in the NS corpus and a higher frequency of 56.7 occurrences in the NNS corpus. The second most frequent orientation is implicit objective $(\mathrm{NS}=24, \mathrm{NNS}=14.3$; epistemic adjectives, adverbs, nouns, and verbs). The third most common category is explicit objective (e.g., The findings suggest that ... ), with 3.1 instances in NS and 4.7 instances in NNS corpora. 
Finally, this is followed by explicit subjective devices (NS $=1.3, \mathrm{NNS}=1.5$; e.g., We conclude that ... ).

\section{Detailed Analysis}

Implicit Subjective Resources

The implicit subjective category was the most common category in the two corpora. The NNS writers relied on modal verbs to a larger extent than did the NS: $73 \%$ vs. $65 \%$, respectively. The cases provided in Example (1) are taken from the two corpora, illustrating the use of modal verbs to express epistemic confidence.

(1) "Giving these students an opportunity to use their prior knowledge might then have been a bridge to better understanding." (NS)

"Such issues can become so problematic that many students may feel unable to continue with a science career." (NNS)

In these examples, the writers used the hedging devices might and may to minimize their level of confidence in their opinions. Thus, the NS's sentence conveys the writer's opinion about the usefulness of students' prior knowledge but simultaneously hedges the statement to cater to opposing opinions. To demonstrate the importance of modal verbs in the present data, Table 6 presents the top ten markers in the two corpora. The top-ten lists consist overwhelmingly of modal verbs plus the markers often, likely, and possible.

Table 6.

The top ten markers in the data

\begin{tabular}{llll|lll}
\hline & \multicolumn{2}{c|}{ NS } & \multicolumn{2}{c}{ NNS } \\
\cline { 2 - 7 } Rank & Marker & $\begin{array}{c}\text { Raw } \\
\text { Freq. }\end{array}$ & $\boldsymbol{f n}$ & can & $\begin{array}{c}\text { Raw } \\
\text { Freq. }\end{array}$ & \multicolumn{2}{c}{$\mathbf{n}$} \\
\hline $\mathbf{1}$ & may & 350 & 13.9 & should & 211 & 16.3 \\
\hline $\mathbf{2}$ & can & 329 & 13.1 & may & 138 & 8.4 \\
\hline $\mathbf{3}$ & might & 148 & 5.8 & will & 74 & 4.5 \\
\hline $\mathbf{4}$ & would & 143 & 5.7 & could & 57 & 3.5 \\
\hline $\mathbf{5}$ & must & 135 & 5.3 & might & 55 & 3.3 \\
\hline $\mathbf{6}$ & often & 124 & 4.9 & must & 53 & 3.2 \\
\hline $\mathbf{7}$ & could & 115 & 4.5 & would & 51 & 3.1 \\
\hline $\mathbf{9}$ & likely & 105 & 4.1 & about & 27 & 1.6 \\
\hline $\mathbf{1 0}$ & should & 63 & 2.5 & possible & 23 & 1.4 \\
\hline
\end{tabular}

As shown in Table 6, may is the most frequently used epistemic marker in the NS corpus. This is consistent with the literature reporting that may is the most common epistemic device in academic discourse (Hyland, 1998; Salager-Meyer, 1994). However, the modal may is the third most frequent marker in the NNS corpus, which uses can as the most frequent modal resource. There are also differences between the two groups in terms of values of modal verbs, as shown in Table 7.

Table 7.

Values of modal verbs used in the corpora (per 10,000 words)

\begin{tabular}{lllllll}
\hline & \multicolumn{2}{c}{ Low } & \multicolumn{2}{c}{ Median } & \multicolumn{2}{c}{ High } \\
\cline { 2 - 7 } & $f \mathrm{n}$ & $\%$ & $f \mathrm{n}$ & $\%$ & $f \mathrm{n}$ & $\%$ \\
\hline NS & 16.1 & $30 \%$ & 29.6 & $55.2 \%$ & 7.8 & $14.6 \%$ \\
\hline NNS & 10 & $17.6 \%$ & 38.5 & $67.8 \%$ & 8.2 & $14.5 \%$ \\
\hline
\end{tabular}

Table 7 shows that median-value markers (i.e., may, can, should, ought to) are more common than low-value markers (i.e., might, would, could) and high-value markers (i.e., must, will, have to) in the two corpora. However, the frequency of low-value modal verbs is larger in 
the NS corpus $(\mathrm{N}=16.1)$ than in the NNS corpus $(\mathrm{N}=10)$. On the other hand, the frequency of median-value modal verbs is larger in the NNS corpus $(\mathrm{N}=38.5)$ than in that of the NS writers $(\mathrm{N}=29.6)$.

In relation to the expression of obligation with deontic modality, it is clear in Table 6 that, when NS writers wish to express obligations, they use the high-value marker must (frequency $=5.3$ ) rather than the median-value marker should (frequency $=2.5$ ), as illustrated by Example (2).

(2)

"the policies and procedures within the organization must free participants from mundane and trivial tasks." (NS)

"All institutions ... should also feel a responsibility for adding to what we know." (NS)

Expressions of advice are even more frequent in the NNS discourse. Table 6 shows a remarkably higher predominance of should in NNS texts $(\mathrm{N}=12.9)$ as compared to NS texts $(\mathrm{N}=2.5)$. This marker is the second marker in the NNS top-ten list, and it alone accounts for $16.7 \%$ of all modality markers used in the NNS corpus compared to only $3 \%$ in the case of the NS corpus.

\section{Implicit Objective Resources}

The largest difference between the two corpora lies in the use of implicit objective resources - adjectives, adverbs, nouns, and verbs. The NS writers used almost twice as many implicit objective resources $(\mathrm{N}=24)$ as the NNS writers $(\mathrm{N}=14.3)$. Table 8 shows that all grammatical categories have a higher frequency in the NS than in the NNS corpus.

Table. 8

Implicit objective resources in the data (per 10,000 words)

\begin{tabular}{lllll}
\hline \multicolumn{5}{c}{ NS } \\
\hline & Low & Median & High & Total \\
\hline Adj. & 1.2 & 4.4 & 1.5 & 7.1 \\
\hline Adv. & 2.9 & 7.6 & 2.4 & 12.9 \\
\hline Nouns & 0.2 & 0.6 & 2 & 2.8 \\
\hline Verbs & 0.7 & 0 & 0 & 0.7 \\
\hline Total & 5 & 12.6 & 5.9 & 23.5 \\
& $(21 \%)$ & $(53 \%)$ & $(25 \%)$ & \\
\hline
\end{tabular}

\begin{tabular}{lllll}
\hline \multicolumn{5}{c}{ NNS } \\
\hline & Low & Median & High & Total \\
\hline Adj. & 1.4 & 1 & 1.5 & 3.9 \\
\hline Adv. & 3 & 2.5 & 1.6 & 7.1 \\
\hline Nouns & 0.1 & 0.3 & 2.2 & 2.6 \\
\hline Verbs & 0.4 & 0 & 0 & 0.4 \\
\hline Total & 4.9 & 3.8 & 5.3 & 14 \\
& $(35 \%)$ & $(27 \%)$ & $(37 \%)$ & \\
\hline
\end{tabular}

Within the implicit objective category, the two corpora differed, particularly in the frequency of epistemic adverbs and adjectives, with these resources being more common in the NS than in the NNS corpus. The differences in this orientation also pertained to the wordings that were preferred by the two groups of writers. Table 9 provides the lists of the top ten implicit objective markers in the data.

Table. 9

Top ten implicit objective resources in the data

\begin{tabular}{clll|lcl}
\hline & & \multicolumn{2}{c|}{ NS } & \multicolumn{3}{c}{ NNS } \\
\cline { 2 - 7 } Rank & Marker & Raw Freq. & $f \mathrm{n}$ & Marker & Raw Freq. & $f \mathrm{n}$ \\
\hline 1 & often & 124 & 4.9 & about & 27 & 1.6 \\
\hline 2 & likely & 105 & 4.1 & almost & 21 & 1.2 \\
\hline 3 & about & 39 & 1.5 & often & 17 & 1 \\
\hline 4 & evidence & 34 & 1.3 & fact & 15 & 0.9 \\
\hline 5 & possible & 32 & 1.2 & evidence & 13 & 0.8 \\
\hline 6 & perhaps & 30 & 1.1 & usually & 12 & 0.7 \\
\hline 7 & almost & 28 & 1.1 & clear & 11 & 0.6 \\
\hline 8 & indeed & 25 & 0.9 & clearly & 8 & 0.4 \\
\hline 9 & potentially & 22 & 0.8 & always & 5 & 0.3 \\
\hline 10 & clear & 18 & 0.7 & &
\end{tabular}


As Table 9 shows, the most frequent implicit objective resources in the NS corpus were the median-value adverb often and the adjective likely, with 4.9 and 4.1 occurrences, respectively. However, often and likely occurred much less frequently in the NNS list, occupying the third and fourth positions with a frequency that is almost four times smaller, 1.0 and 0.9, respectively. On the other hand, the top two markers in the NNS corpus were about and almost. Example (3) instantiates the use of these resources in the data.

"professional development providers often lack in-depth knowledge of content" (NS)

“Almost all students (96.8\%) also did not write any reason for their responses." (NNS)

Writers use often to make statements indefinite and to distinguish them from facts. They also use likely to judge the probability of a certain situation to which they are not completely committed. For example, the NS sentence in (3) conveys a negative judgment (Martin \& White, 2005) of the competence shown by professional development providers with the claim that this competence is not deep enough. But the writer here is careful to weaken the claim with often to soften the criticism and to allow readers the freedom to dispute it in case they do find the mentioned competence deep enough. On the other hand, the NNS writer in (3) qualifies a numerical statement with almost. The rhetorical effect here does not involve providing space for readers to dispute the statement but is meant to temper the generalization to make the statement more accurate.

Additionally, the NS top-ten list included the three boosting devices evidence, indeed, and clear, while the boosters figuring in the NNS top-ten list were more in terms of types: evidence, clear, clearly, and always. This is exemplified in (4).

"Indeed, one outcome of the great success of Situated Learning is that ..."

"The students always showed engagement in group work" (NNS)

The use of the booster indeed assisted the NS writer in (4) in reinforcing the truth value of the proposition and in conferring a higher degree of commitment to the truth of the positive evaluation of situated learning. Similarly, the NNS writer's positive appreciation of group work as a teaching methodology is boosted with always to underlie the writer's maximal conviction concerning the effectiveness of this methodology.

Finally, in contrast to the frequency of median-value and high-value implicit objective devices, the low-value evidential verbs seem and appear were not among the top ten markers in the two corpora. Each occurred only nine times in the NS corpus. Similarly, the NNS corpus used appear only six times with only one incidence of seem.

\section{Explicit Subjective Resources}

The explicit subjective orientation had the lowest frequency among the other three orientations in both corpora. In terms of between-group differences, the NNS writers used more explicit subjective constructions $(\mathrm{N}=1.5)$ than did NS writers $(\mathrm{N}=1.3)$, as shown in Table 10.

Table 10.

Explicit subjective resources in the data (per 10,000 words)

\begin{tabular}{llllllll}
\hline & Low & & Median & & High & Total \\
\cline { 2 - 7 } & $\boldsymbol{f n}$ & $\mathbf{\%}$ & $\boldsymbol{f n}$ & $\mathbf{\%}$ & $\boldsymbol{f n}$ & $\boldsymbol{\%}$ & \\
\hline NS & 0.03 & $3 \%$ & 0.07 & $6 \%$ & 1.2 & $93 \%$ & 1.3 \\
\hline NNS & 0.1 & $11.5 \%$ & 0.3 & $19 \%$ & 1.1 & $69 \%$ & 1.5 \\
\hline
\end{tabular}


Also, as noted in Table 10, explicit subjective constructions incorporating low- and median-value (possibility and probability) markers, namely, suggest/hypothesize/propose, were extremely infrequent, with only one or no occurrence in the two corpora. Moreover, the possibility/probability cognition verbs think, feel, suppose, speculate, deduce, infer, and assume never appeared in explicit subjective constructions in the NS corpus, again signaling the lack of reliance on this category as an epistemic resource for claim qualification.

On the other hand, the two groups of writers used this orientation with high-value certainty markers: $93 \%$ for NS and $69 \%$ for the NNS corpus. The markers that accounted for these higher percentages are the epistemic verbs find and conclude, which were used in constructions referring to the researchers as the subject of the sentence, as in Example (5).

(5) "We found high student participation across the videos." (NS)

"We concluded that students have difficulty in differentiating between direct and indirect division in the animal cells." (NNS)

Explicit Objective Resources

Table 11.

Explicit objective resources in the data (per 10,000 words)

\begin{tabular}{llllllll}
\hline & Low & \multicolumn{3}{c}{ Median } & High & Total \\
\cline { 2 - 7 } & $\boldsymbol{f n}$ & \% & $\boldsymbol{f n}$ & $\boldsymbol{\%}$ & $\boldsymbol{f n}$ & $\boldsymbol{\%}$ & \\
\hline NS & 0.9 & $29 \%$ & 0.8 & $25 \%$ & 1.3 & $41 \%$ & 3.1 \\
\hline NNS & 0.6 & $12.7 \%$ & 0.9 & $19 \%$ & 3.1 & $65 \%$ & 4.7 \\
\hline
\end{tabular}

Table 11 shows that the NS writers used high-value explicit objective constructions $(41 \%)$ more often than low- $(29 \%)$ and median-value resources $(25 \%)$. A number of high-value markers account for the higher percentage. They include the boosting verbs reveal, confirm, establish, demonstrate, show, find, and provide evidence. These verbs were higher in frequency than tentative verbs, such as suggest, propose, imply, and indicate. Moreover, the NNS writers' use of high-value objective expressions (3.1) was even higher than that of NS writers (1.3), indicating their stronger level of certainty about the validity of their findings and conclusions. The most frequent high-value explicit objective expression used by the NNS writers was it is clear that. The use of this category by both groups can be exemplified in (6):

(6) "This study demonstrates that a short-term placement like the UEFP can simultaneously cultivate culturally responsible candidates." (NS)

"Our study finds evidence that prospective teachers have stronger average preferences." (NS)

"It is clear that age somehow had an effect on reading comprehension." (NNS)

"It is clear that Saudi science teachers mainly follow a discovery approach in all research-targeted areas." (NNS)

\section{Discussion}

The present study aimed to describe how epistemic modality is used to express certainty and subjectivity in English education research by native and non-native expert writers. The forgoing analysis revealed patterns of use of modality by the two groups of writers that can be interpreted in terms of the influence of register-, discipline-, and culture-specific factors. To begin, the present study provides further evidence of the importance of epistemic modality as a 
rhetorical device that writers of research papers uniformly employ (Hyland, 1994). The predominance of hedges (low- and median-value markers combined) over boosters (high-value markers) in the present education research also conforms to similar findings in other disciplines (Hyland, 1994; Hyland \& Milton, 1997; Yang et al., 2015).

However, the overall register-necessitated similarity between the present discourse and other academic disciplines is also accompanied by distinctive characteristics that belong particularly to the present data and that seem to be underpinned by disciplinary proclivities. These characteristics are related to both values and orientations of modality.

\section{Values of modality in NS discourse}

In terms of values of modality, the finer-grained analysis that relies on the differentiation between the two degrees of hedging strength (i.e., low vs. median) suggests that the present discourse was moderately confident in its authorial stance rather than being completely cautious and reserved. Although the present writers used more hedges than boosters to conform to rhetorical norms of academic discourse, they simultaneously employed the more confident end of the uncertainty continuum to create sufficiently convincing arguments. There are a number of indicators that point to this characterization.

Overall, the NS writers preferred the following distribution of values, from most frequently to least frequently occurring: median (probability) $>$ low (possibility) $>$ high (certainty). This stance contrasts with another more hedged stance that relies on a larger degree of hesitancy and doubt by employing the pattern possibility > probability > certainty, and that has been found to have been employed in other academic disciplines, such as medical discourse (e.g., Yang et al., 2015). By semantic definition, the greater use of probability than possibility in the present data signaled a higher level of authorial commitment and indicated the writers' consideration of their claims as having a median (not low) likelihood of being true.

The infrequency of specific hedging devises also points to the present writers' tendency to sound reasonably confident. For one thing, the tentative evidentials seem and appear, which have been traditionally reported as having a high frequency in academic corpora (Hyland, 1994), were extremely infrequent in the present data. As Hyland (1994, p. 249) suggests, writers use these words when they want to report their findings and simltaneously highlight the speculative nature of their propositions. Their scarcity in the present data signifies the writers' adoption of a confident stance in the presentation of their findings. Also, explicit subjective constructions incorporating low- and median-value markers, namely, suggest, hypothesize, propose, indicate, think, feel, suppose, speculate, deduce, infer, and assume, were extremely infrequent with only one or no occurrence in the two corpora. This is contrary to Crosthwaite et al. (2017), who found that indicate and suggest occurred frequently in professional discourse to hedge authors' own claims, and to Hyland (1998), who found that believe, feel, and suspect are important devices in the social sciences.

On the other hand, in the presentation of their findings and conclusions, the present NS writers tended to project a confident tone by using boosting devices, such as find, show, reveal, demonstrate, establish, and give evidence. Martin and White (2005) conceptualize these devices as realizing the semantic category endorsement. This category presents propositions as being completely valid and encodes the writer as adopting with them. This, in turn, contracts the dialogic space for alternative opinions and thus construes the readers, too, as being in alignment with the strong and credible textual voice.

Commitment to the validity of claims was also reflected in NS writers' frequent use of the high-value deontic modal must, which figured as one of the top ten devices in their discourse. Deontic modality refers to a writer's intrusion into the speech event to impose obligations and to give permission (Piqué-Angordans et al. 2002). The rhetorical function involves the construal of the writer as one who is in a position of power and responsibility to impose their specific point of view and to provide directions on how participants ought to 
behave. Therefore, deontic modality is often associated with an assertive authorial tone (PiquéAngordans et al., 2002). In fact, the frequency of this modality in education research is understandable given this field's aims "to speak to and be useable by practitioners" (Yates, 2004, p. 13) and to provide knowledge needed "to make informed decisions about educational practice [and] policy on programs" (Anderson \& Arsenault, 1998, p. 4). However, what is interesting from a linguistic perspective is the present writers' preference to use the strongest form of this kind of modality (i.e., must), a practice that reflected their strong confidence in the validity of the solutions that they offered.

\section{Orientation of modality in NS discourse}

In terms of orientations of modality, writers attempted to build convincing arguments by sounding detached and impersonal. Writers objectified their attitudes through the use of implicit and objective modality orientations. First, the writers' use of implicit devices, both subjective and objective, was much more common than their use of explicit modality. Also, within the explicit orientation, the writers avoided the use of the subjective option (I think that) and employed more objective constructions (The findings show that). Through this pattern, writers concealed their responsibility for the presented convictions and "experientialized" (Thompson, 2014 , p. 73) their viewpoint by making it appear as an objective property of the described situation. This also suppressed the writers' textual presence and rendered the discourse more fact-oriented (Yang et al., 2015).

Hyland (1999, p. 364) describes research in hard sciences in a similar way by maintaining that, in hard sciences, "the authority of the individual is subordinate to the authority of the text". This contrasts with research in the humanities and social sciences in which writers are more likely to emphasize their subjectivity and their personal responsibility for the expressed judgment (Takimoto, 2015). The tendency of the present writers to assume an epistemic stance that is more akin to the one adopted in the hard sciences than in the social sciences points to the discipline-specific nature of the rhetorical practices in education research that make texts appear to readers as more objective and fact-oriented than interpretative and personal.

\section{Epistemic modality in NNS discourse}

The variance in frequencies and categories of the employed epistemic options resulted in what can be described as a less interactional and even more confident style in the NNS discourse. The NNS writers used an overall smaller number of epistemic markers. This finding, whereby NNS texts were less interactional and interpersonal due to including less epistemic modality than NS discourse, is consistent with previous research $(\mathrm{Hu} \& \mathrm{Li}, 2015$; Akbas \& Hardman, 2018; Al-Mudhaffari et al., 2020). Hu and Li (2015, p. 21) observed that, "The direct and unqualified writing . . . seems to pertain to most L2 writers regardless of their L1 backgrounds". Based on the present findings, one can also add that L2 writers are likely to have this tendency despite their level of language proficiency, since the present EFL writers possess a high level of linguistic proficiency and still prefer a less qualified style than that employed by their native counterparts.

Another indicator of NNS writers' higher level of confidence is their pattern of values of modality, which proved to be as follows: median (probability) > high (certainty) > low (possibility). This pattern, which includes more certainty than possibility, signifies the expression of firmer assertions and a greater level of confidence. This higher level of confidence can be attributed to the Arab writers' cultural tendency toward assertion and exaggeration through the use of emphatics, boosters, and monologism as validated by previous research (e.g., El-Seidi, 2000; Abu Rass, 2011; Alramadan, 2020b; Mudhaffari et al., 2020).

In terms of the use of individual markers, the overwhelming frequency of the deontic modal should also points to the confident character of the NNS discourse. Should was the 
second most frequently used device in the NNS corpus and it alone had a higher frequency than the two deontic modals must and should in the NS discourse. This indicates that the NNS writers generalized more extensively from their findings. This could result from the NNS writers' higher degree of confidence in the robustness of their studies and the validity of the recommendations emerging from them. Alternatively, the higher number of recommendations in the NNS texts could be a result of these writers' emphasis on the practical and situational, rather than conceptual, aspects of research, which led their discourse to be more "agentoriented" (Collins, 2009, p. 22). The Arab writers' emphasis on topic-oriented rather than research-oriented ideational fields of discourse has already been attested to in the discipline of applied linguistics (Alramadan, 2020a). The validation of this kind of contrast, however, requires future genre-based research to investigate not only the interpersonal but also the ideational metafunction of education research (Halliday and Matthiessen, 2004).

A number of other differences occurred between the NS and NNS texts that resulted in a less idiomatic use of the modality system by the NNS writers. These included the over/underuse of modality options. Specifically, the NNS writers overused the implicit subjective orientation and underused the implicit objective orientation. The difference between the frequencies of the two orientations in the NNS discourse (four times) was much higher than the difference in the NS discourse (two times). Holmes (1988) maintained that epistemic grammatical classes constitute around $27 \%$ of the epistemic resources used in written discourse. The present NS writers' percentage of these categories $(29 \%)$ is close to the figure reported by Holmes, whereas the NNS writers' percentage (18\%) is remarkably smaller. The heavy use of modal auxiliaries at the expense of other epistemic resources was corroborated by L2 research in relation to both Arab (Al-Mudhaffari et al., 2020) and non-Arab writers (Hu \& Li, 2015).

The overuse of modal operators in the NNS corpus not only decreased the use of other objective epistemic options, but also heightened the degree of textual subjectivity. Modal operators are subjective resources, as they present the proposition as "grounded in the individual subjectivity of the authorial voice" (Martin \& White, 2005, p. 98). Furthermore, the subjectivity in this corpus was also higher because NNS writers employed more explicit subjective constructions (We found/conclude) than did the NS writers. The rhetorical effect includes a higher degree of personal presence.

Finally, the two corpora varied in the selection of different epistemic wordings from within the same semantic categories. The most notable difference relates to the use of implicit objective devices. In this category, the most frequent words in the NS corpus were often and likely, which were much less frequent in the NNS corpus. On the other hand, the most frequent implicit objective devices in the NNS corpus were almost and about. The difference between these two pairs of devices is basically a difference in the reader-engagement effect that they engender, with the first pair being more reader-oriented. The hedges often and likely are among what Martin and White (2005) call entertain resources, which they define as "those wordings by which the authorial voice indicates that its position is but one of a number of possible positions and thereby, to greater or lesser degrees, makes dialogic space for those possibilities" (Martin \& White, 2005, p. 104). The NS writers relied on these devices to make their statements tentative and to expand dialogic space for other value positions. In contrast, the two expressions almost and about are called approximators (Salager-Meyer, 1994), attribute hedges (Hyland, 1998), or devices for softening the focus of the expressed proposition (Martin \& White, 2005). In the data, writers used them in propositions containing numerical information (e.g., about $67.2 \%$ of the sample), not to open up dialogic space but to highlight their precision in providing accurate measurements. These devices are therefore accuracy-oriented. This means that the Arab writers did not avail themselves to the exploitation of the same reader-oriented resources that are conventionally used by the native writers of education research. 


\section{CONCLUSION}

The present study examined how English-speaking and Arabic-speaking L2 writers of education research use epistemic modality to qualify their claims and to construe stances toward their texts and their readers. The findings substantiated the "pragmatically sophisticated" (Wishnoff, 2000, p. 122) nature of epistemic stance construction. The writers used an overall higher frequency of hedges than boosters, but at the same time managed, through employment and avoidance of certain resources, to express a moderate degree of confidence to serve the needs and purposes of the disciplinary audience that is made up of not only peer researchers but also practitioners looking for validated solutions to put into actual action. The findings also demonstrated the impact of discipline as a contextual determinant of the rhetorical nature of academic discourse. The epistemic style displayed in the current discourse, in some respects, equated it with research in the hard sciences and, in other respects, distinguished it from research in the social sciences, to which it is supposed to belong. From linguistic and pedagogical perspectives, then, the study points to the limited usefulness of such broad categorizations as hard vs. humanities/social sciences and of the assumption that a particular text will display certain rhetorical features only because of its affiliation with one of these two broad categories. Finally, the study revealed cross-cultural aspects in the use of epistemic values, orientations, and wordings that were not completely overcome by writers with high linguistic proficiency, but were rather reminiscent of features attributed by the literature to other L2 writers/learners at lower levels of proficiency.

The limitations of the present study include the focus on discourse that is produced in an extended 10-year time span. Therefore, there might have been some diachronic characteristics that affected the patterns identified. Also, for convenience purposes, the sampling of the native corpus was restricted to a single scientific journal. This may affect the generalizability of the findings as the identified patterns may have been a product of the particular linguistic specifications expected from authors by the selected journal. Finally, the holistic entire-article approach that is adopted by the study many have missed important genre-related characterizations pertaining to the use of epistemic modality in specific sections of the research article.

Notwithstanding these limitations, the present study provides important implications for Arab EFL academic writers as well as for English for Academic Purposes pedagogy in Arab higher-education institutions. These writers should be made aware of the full range of resources that are available in the system of epistemic modality. They should also be sensitized to the discursive norms of the research paper by being introduced to the notions of values and orientations of modality in order to enable them to optimally temper the degrees of confidence and subjectivity in their writing. Writers need to know the rhetorical repercussions resulting from the use of each modality configuration to produce an epistemic stance that is persuasively effective from the perspective of the international discourse community.

\section{REFERENCES}

Abu-Rass, R. (2011). Cultural transfer as an obstacle for writing well in English: The case of Arabic speakers writing in English. English Language Teaching, 4(2): 206-212.

Akbas, E. \& Hardman, J. (2018). Strengthening or weakening claims in academic knowledge construction: A comparative study of hedges and boosters in postgraduate academic writing. Educational Sciences: Theory \& Practice, 18(4): 831-859.

Al-Mudhaffari, M., Hussin, S. \& HoAbdullah, I. (2020). Interactional strategies in L2 writing: An exploration of hedging and boosting strategies in applied linguistics research articles. International Journal of Arabic-English Studies, 20(1): 171-186. 
Alotaibi, H. (2015). Metadiscourse in Arabic and English Research Article Abstracts. World Journal of English Language, 5(2): 1-8.

Alramadan, M. M. (2020a). Authorial Stance in English, Arabic and EFL Applied Linguistics Research: An Appraisal Study. Asiatic: IIUM Journal of English Language and Literature, 14(1), 189-216.

Alramadan, M. M. (2020b). The Use of Engagement Resources in English, Arabic, and EFL Applied Linguistics Research: A Contrastive Study Within an Appraisal Theoretic Perspective. In E. El-Sadig \& T. Drid (eds.), Teaching Academic Writing as a DisciplineSpecific Skill in Higher Education, 23-54. IGI Global.

Anderson, G. \& Arsenault, N. (1998). Fundamentals of educational research. Taylor and Francis Group.

Butler, C. (1990). Qualifications in science: Modal meanings in scientific texts. In W. Nash (ed.), The writing scholar: Studies in academic discourse, 137-170. New York: Sage.

Collins, P. (2009). Modals and quasi-modals in English. New York: Rodopi.

Crosthwaite, P. Cheung, L. \& Jiang, F. (2017). Writing with attitude: Stance expression in learner and professional dentistry research reports. English for Specific Purposes, 46: $107-123$.

Doğan, Z. N. \& Akbaş, E. (2021). An exploratory study of epistemic stance in results and discussion sections of medical research articles. Atatürk Üniversitesi Sosyal Bilimler Enstitüsü Dergisi, 25(3), 252-270.

El-Seidi, M. (2000). Metadiscourse in English and Arabic argumentative writing: A crosslinguistic study of texts written by American and Egyptian university students. In Z. Ibrahim, N. Kassabgy \& S. Aydelott (eds.), Diversity in language: Contrastive studies in English and Arabic theoretical and applied linguistics, 111-126. Cairo and New York: The American University in Cairo Press.

Farnia, M., \& Gerami, S. (2021). Comparative Study of Interactional Metadiscourse Markers in the Discussion Section of Soft and Hard Science Research Articles: Hedges and Boosters in Focus. Jordan Journal of Modern Languages and Literatures, 13(2), 263280.

Flowerdew, J. (2002). Academic discourse. London: Pearson Education.

Halliday, M. A. K. \& Matthiessen, C. (2004). An introduction to functional grammar. London: Hodder Education.

Holmes, J. (1982). Expressing doubt and certainty in English. RELC Journal, 13(2): 9-28.

Holmes, J. (1984). Modifying illocutionary force. Journal of Pragmatics, 8(3): 345-365.

Holmes, J. (1988). Doubt and certainty in ESL textbooks. Applied Linguistics, 9(1): 21-44.

$\mathrm{Hu}, \mathrm{C} . \& \mathrm{Li}, \mathrm{X}$. (2015). Epistemic modality in the argumentative essays of Chinese EFL learners. English Language Teaching, 8(6): 20-31.

Hyland, K. (1994). Hedging in academic writing and EAP textbooks. English for Specific Purposes, 13(3): 239-256.

Hyland, K. (1996). Writing without conviction? Hedging in science research articles. Applied linguistics, 17(4): 433-454.

Hyland, K. (1998). Boosting, hedging and the negotiation of academic knowledge. TextInterdisciplinary Journal for the Study of Discourse, 18(3): 349-382.

Hyland, K. \& Milton. J. (1997). Qualification and certainty in L1 and L2 students' writing. Journal of Second Language Writing, 6(2): 183-205.

Koutsantoni, D. (2005). Certainty across cultures: A comparison of the degree of certainty expressed by Greek and English speaking scientific authors. Intercultural Pragmatics, 2(2): 121-149. 
Lakoff, G. (1972). Hedges: A study in meaning criteria and the logic of fuzzy concept. Chicago Linguistics Society Papers, 8: 183-228.

Lyons, J. (1977). Semantics. Cambridge: Cambridge University Press.

Martin, J. R., \& White, P. R. (2005). The language of evaluation. London: Palgrave Macmillan.

Mirzapour, F. \& Mahand, M. (2012). Hedges and boosters in native and non-native library and information and computer science research articles. The Southeast Asian Journal of English Language Studies, 18(2): 119-128.

Myers, G. (1989). The pragmatics of politeness in scientific articles. Applied Linguistics, 10(1): $1-35$.

Piqué-Angordans, J., Posteguillo, S. \& Andreu-Besó, V. (2002). Epistemic and deontic modality: a linguistic indicator of disciplinary variation in academic English. LSP and Professional Communication, 2(2): 49-65.

Salager-Meyer, F. (1994). Hedges and textual communicative function in medical English written discourse. English for Special Purposes, 13(2): 149-170.

Sultan, A. (2011). A contrastive study of metadiscourse in English and Arabic linguistics research articles. Acta Linguistica, 5(1): 28-41.

Swales, J. (1990). Genre analysis: English in academic and research settings. Cambridge: Cambridge University Press.

Takimoto, M. (2015). A corpus-based analysis of hedges and boosters in English academic articles. Indonesian Journal of Applied Linguistics, 5(1): 95-105.

Thompson, G. (2014). Introducing functional grammar. London and New York: Routledge.

Vold, E. (2006). Epistemic modality markers in research articles: a cross-linguistic and crossdisciplinary study. International Journal of Applied Linguistics, 16(1): 61-87.

Wishnoff, J. (2000). Hedging your bets: L2 learners' acquisition of pragmatic devices in academic writing and computer-mediated discourse. Second Language Studies, 19(1): $119-148$.

Yang, A., Zheng, S. \& Ge, G. (2015). Epistemic modality in English-medium medical research articles: A systemic functional perspective. English for Specific Purposes, 38: 1-10.

Yates, L. (2004). What does good educational research look like? New York: Open University Press. 